\title{
Emodin Ameliorates High Glucose Induced-Podocyte Epithelial-Mesenchymal Transition In-Vitro and In-Vivo
}

\author{
Tingfang Chen ${ }^{\mathrm{a}} \quad$ Li Yang Zheng $^{\mathrm{b}} \quad$ Wenzhen Xiao $^{\mathrm{a}} \quad$ Dingkun Gui $^{\mathrm{b}} \quad$ Xiaoxia Wang ${ }^{\mathrm{b}}$ \\ Niansong Wang ${ }^{b}$
}

aDepartment of Medicine, Soochow University, Suzhou, bDepartment of Nephrology and Rheumatology, Shanghai Jiaotong University Affiliated Sixth People's Hospital, Shanghai, PR China

\section{Key Words}

Emodin - Podocyte epithelial-mesenchymal transition - Integrin-linked kinase - Desmin • Nephrin • Diabetic kidney disease

\begin{abstract}
Background: Epithelial-to-mesenchymal transition (EMT) is a potential pathway leading to podocyte depletion and proteinuria in diabetic kidney disease (DKD). Here, we investigated the protective effects of Emodin (EMO) on high glucose (HG) induced-podocyte EMT invitro and in-vivo. Methods: Conditionally immortalized mouse podocytes were exposed to $\mathrm{HG}$ with $30 \mu \mathrm{g} / \mathrm{ml}$ of EMO and $1 \mu \mathrm{mol} / \mathrm{ml}$ of integrin-linked kinase (ILK) inhibitor QLT0267 for $24 \mathrm{~h}$. Streptozotocin (STZ)-induced diabetic rats were treated with EMO at $20 \mathrm{mg} \cdot \mathrm{kg}^{-1} \cdot \mathrm{d}^{-1}$ and QLT0267 at $10 \mathrm{mg} \cdot \mathrm{kg}^{-1} \cdot \mathrm{w}^{-1}$ p.o., for 12 weeks. Albuminuria and blood glucose level were measured. Immunohistochemistry, immunofluorescence, western blotting and real-time PCR were used to detect expression of ILK, the epithelial marker of nephrin and the mesenchymal marker of desmin in-vitro and in-vivo. Results: HG increased podocyte ILK and desmin expression while decreased nephrin expression. However, EMO significantly inhibited ILK and desmin expression and partially restored nephrin expression in HG-stimulated podocytes. These in-vitro observations were further confirmed in-vivo. Treatment with EMO for 12 weeks attenuated albuminuria, renal histopathology and podocyte foot process effacement in diabetic rats. EMO also repressed renal ILK and desmin expression, preserved nephrin expression, as well as ameliorated albuminuria in STZ-induced diabetic rats. Conclusion: EMO ameliorated glucose-induced EMT and subsequent podocyte dysfunction partly through ILK and desmin inhibition as well as nephrin upregulatiotion, which might provide a potential novel therapeutic option for DKD.
\end{abstract}

Dingkun Gui and Niansong Wang

KARGER 125
Department of Nephrology and Rheumatology, Shanghai Jiaotong University Affiliated Sixth People's Hospital, 600 Yishan Road, Shanghai, 200233(China)

E-Mail guidingkun@sina.com.cn and E-Mail wangniansong2012@163.com 


\section{Introduction}

Diabetic kidney disease (DKD) is a serious microvascular complication of type 1 and 2 diabetes and a major cause of end-stage renal disease (ESRD) in the developed countries [1]. Therefore, there is an urgent need for the development of novel approaches for treatment of DKD. Podocytes are terminally differentiated epithelial cells that reside on the glomerular basement membrane (GBM) outside the glomerular capillaries. The clinical study demonstrated that podocyte loss contributed to the progression of DKD [2]. Yamaguchi et al. proposed a new explanation for podocyte loss through an epithelial to mesenchymal transition (EMT) in patients with DKD [3]. For many common glomerular diseases such as DKD, EMT could be a potential pathway contributing to podocyte dysfunction,proteinuria, and glomerulosclerosis [4]. EMT will render podocytes motile and ultimately cause the disruption of the delicate three-dimensional architecture of podocytes, thereby damaging glomerular filtration barrier function [5]. Identification of significant amount of viable podocytes in urine from both animal models of DKD and from patients with DKD suggest that podocyte loss might be caused by reduced podocyte adhesion,which is a potential consequence of EMT $[6,7]$. Thus, podocyte EMT may play an important role in causing podocyte loss, which represents a novel early mechanism leading to DKD.

Emodin (1,3,8-trihydroxy-6-methylanthraquinone) is one of the major components in the root and rhizome of Rheum palmatum such as rhubarb. Recent studies have demostrated that Emodin protected against cisplatin-induced oxidative stress in cultured HEK 293 cells [8] and attenuated hypoxia and LPS-induced intestinal epithelial barrier dysfunction through inhibition of NF- $\kappa$ B and HIF-1 $\alpha$ signaling pathways [9]. Emodin has been reported to protect against diabetic cardiomyopathy by regulating the AKT/GSK-3 $\beta$ signaling pathway in rats [10]. The animal study demonstrated that Emodin ameliorated renal dysfunction through inhibition of activation of p38 MAPK pathway in rats with DKD [11]. However, the protective effects of Emodin on podocyte EMT in rats with DKD have not been investigated yet. This study aimed to investigate the protective effects of Emodin on high glucose induced-podocyte EMT in-vitro and in-vivo, and then provide a potential novel therapeutic option for DKD.

\section{Materials and Methods}

\section{Drug preparation}

Emodin (EMO, chemical structure $\mathrm{C}_{15} \mathrm{H}_{10} \mathrm{O}_{5}$, molecular weight $=270$ ) was purchased from Nanjing Qingze Medical Technology Development Co.,Ltd. (purity>99\%, Nangjing, China). A high-selective, smallmolecule ILK inhibitor QLT-0267 was purchased from Vancouver, BC, Canada. All other chemicals were of analytic grade and were obtained from Sigma (St. Louis, MO).

\section{In-vitro studies: Cell culture and EMO treatment}

Conditionally immortalized mouse podocytes were kindly provided by Dr. Peter Mundel (Department of Medicine, Massachusetts General Hospital and Harvard Medical School) and were conducted as described previously [12]. In brief, podocytes were cultured in RPMI 1640 supplemented with 10\% heat-inactivated fetal calf serum (FCS, Gibco, USA), $100 \mathrm{U} / \mathrm{ml}$ penicillin and $100 \mu \mathrm{g} / \mathrm{ml}$ streptomycin. Cells were grown at $33^{\circ} \mathrm{C}$ in the presence of $10 \mathrm{U} / \mathrm{ml}$ mouse recombinant interferon- $\gamma$ (IFN- $\gamma$, Sigma Chemical Corporation, USA). To induce differentiation, podocytes were maintained at $37^{\circ} \mathrm{C}$ on collagen-coated dishes without IFN- $\gamma$ to allow differentiation for at least 7 days. Differentiated cells were identified by their large arborized shape and by the cell expression of a known differentiation marker synaptopodin [13]. Differentiated podocytes were cultured for $24 \mathrm{~h}$ in RPMI 1640 medium containing $5 \mathrm{mM}$ D-glucose and 1\% FCS before being exposed to various experimental conditions.

The cells were then divided into four groups: (1) normal glucose group (NG) as controls incubated in RPMI 1640 containing 5 mM glucose, (2) high glucose group (HG) incubated in RPMI 1640 containing 30 mM glucose, (3) EMO group incubated in HG medium treated with 30 $\mu \mathrm{g} / \mathrm{ml}$ of EMO for $24 \mathrm{~h}$, (4) QLT0267 group incubated in HG medium treated with $1 \mu \mathrm{mol} / \mathrm{ml}$ of ILK inhibitor QLT0267 for $24 \mathrm{~h}$. All the glucose used in the present study was D-glucose. All experimental groups were cultured in quadruplicate.

\section{KARGER}




\section{Cellular Physiology Cell Physiol Biochem 2015;35:1425-1436

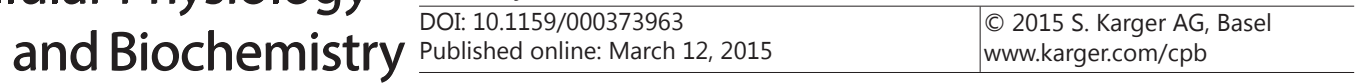 \\ Chen et al.: Emodin Ameliorates Podocyte Epithelial-Mesenchymal Transition}

Animal studies

All the animal procedures were performed in accordance with the "Guide for the Care and Use of Laboratory Animals" published by the National Institutes of Health. All the animal protocols were approved by the Animal Ethics Committee of Shanghai Jiaotong University Affiliated Sixth People's Hospital, Shanghai, PR China. The animal production license number was SCXK (Hu) 2013-0016. Healthy male Sprague-Dawley rats weighing 200 to 220g, purchased from Shanghai Sippr BK Laboratory Animal Co.,Ltd. Shanghai, China, were housed in an air-conditioned room at $23 \pm 1^{\circ} \mathrm{C}$ on a 12 :12-h light-dark cycle. Animals were fed a standard diet and given water ad-libitum. Diabetes was induced in rats by a single intraperitoneal injection of streptozotocin (STZ) dissolved in $0.1 \mathrm{M}$ citrate buffer ( $\mathrm{pH} 4.5$ ) at $60 \mathrm{mg} / \mathrm{kg}$, while the normal control rats received the $0.1 \mathrm{M}$ citrate buffer solution.72 hours after injection of STZ, the blood glucose level was measured from the tail vein. Rats with a blood glucose level over $300 \mathrm{mg} / \mathrm{dl}$ were considered to be diabetic and included in the study. Diabetic rats were then randomly divided into four groups ( $\mathrm{n}=12 /$ each group): (1) STZ-induced diabetic rats (DM); (2) STZ-induced diabetic rats treated with a dose of EMO at $20 \mathrm{mg} \cdot \mathrm{kg}^{-}$ ${ }^{1} \cdot \mathrm{d}^{-1}$ (DM+EMO);(3) STZ-induced diabetic rats treated with a dose of ILK inhibitor,QLT0267 at $10 \mathrm{mg} \cdot \mathrm{kg}^{-1} \cdot \mathrm{d}^{-1}$ (DM+QLT0267); (4) Normal Sprague-Dawley rats were choosed as control (Normal). EMO and QLT0267 were started at 2 weeks after STZ injection and were administered once daily by oral garage for 12 weeks. The normal control (Normal) and diabetic control (DM) rats were treated with the same volume of saline within the same time. At the end of the 12 -week treatment, animals were then killed and the kidneys were harvested immediately.

\section{Morphological studies}

That of the right kidneys was fixed with $10 \%$ buffered formalin and embedded in paraffin for histological evaluation. The kidneys were cut into $4 \mu \mathrm{m}$ sections and stained with hematoxylin and eosin (HE). The sections were then examined by light microscopy in a blind fashion. Glomerular injury was evaluated by mesangial expansion in sections stained with HE. The mesangial area was counted as mesangial expansion, which was determined in 20 consecutive glomeruli from each rat.

To determine the morphological changes in podocytes, electron microscopic morphometric evaluation was performed by routine procedures. Renal cortex samples were cut into $1 \mathrm{~mm}^{3}$ pieces on ice, immediately fixed in $2.5 \%$ glutaraldehyde, and then embedded. Ultrathin sections were examined by electron microscopy. The morphologic assessment was performed independently by two blinded investigators.

\section{Immunohistochemical analysis}

Sections of kidneys from the experimental rats were immunostained for nephrin desmin and ILK (Santa Cruz Biotechnology, Santa Cruz, CA). Immunostaining procedures were performed according to the manufacturer's instructions. Paraffin-embedded sections ( $5 \mu \mathrm{m}$-thick) were deparaffinized with xylene and rehydrated through a descending ethanol gradient. Primary antibodies were diluted in PBS containing 1\% bovine serum albumin (BSA). All antibodies were incubated during 45 minutes at room temperature. The sections incubated with PBS, instead of the primary antibody, served as the negative controls. The amount of nephrin desmin and ILK was analyzed using the Image-Pro P1 uS(IPP) Version 7.0 (Media Cybernetics, USA). In each glomerulus, the percentage of positive area within the glomerular area was calculated. All slides (20 glomeruli per section and $n=6$ per group) were observed independently by two blinded investigators.

\section{Western blotting}

Isolated rat glomeruli or cultured podocytes under different experimental conditions were lysed in lysis buffer. The lysates were clarified by centrifugation at 12,000 × g for $15 \mathrm{~min}$. Protein estimation was performed by Coomassie brilliant blue. The tissue protein was separated by sodium dodecyl sulfate (SDS)/polyacrylamide gel electrophoresis and transferred to a polyvinylidene difluoride membrane. The membranes were blocked for $1 \mathrm{~h}$ at room temperature with PBS containing 5\% skim milk. Membranes were then incubated overnight with nephrin,desmin and ILK antibodies (Santa Cruz Biotechnology, Santa Cruz, CA); Negative controls were performed without primary antibody. After washing, the secondary antibody was added and incubated $1 \mathrm{~h}$ at room temperature. Chemiluminescence detection was performed with the $\mathrm{KC}^{\mathrm{TM}}$ Detection kit (KC-420, KangChen Biotechnology, Shanghai). Equality of loading was ensured by using a monoclonal antibody to $\beta$-actin (Santa Cruz Biotechnology, Santa Cruz, CA). Densitometric quantitation was performed using a Bio-Rad VersaDoc imaging system model 5000 with Bio-Rad Quantity One software. Protein expression was quantified as the ratio of specific band to $\beta$-actin. 


\section{Cellular Physiology Cell Physiol Biochem 2015;35:1425-1436 \begin{tabular}{l|l} 
and Biochemistry Published online: March 12, 2015 & $\begin{array}{l}\text { C 2015 S. Karger AG, Basel } \\
\text { www.karger.com/cpb }\end{array}$ \\
\hline
\end{tabular} \\ Chen et al.: Emodin Ameliorates Podocyte Epithelial-Mesenchymal Transition}

Real-time quantitative RT-PCR

Total RNA was extracted from samples of isolated rat glomeruli or cultured podocytes by the Trizol reagent (Invitrogen, Carlsbad, CA) and treated with RNase-free DNase (Invitrogen). Then, $2 \mu \mathrm{g}$ of total RNA was reverse transcribed using the SuperScript RT kit according to the protocol recommended by the manufacturer (Invitrogen, Carlsbad, CA). The following sequence-specific primers for mouse podocytes were used: nephrin: sense 5'- CGA AGG TGC GCA TAG TTC CG -3'and anti-sense 5'- ACC ACG GGC ATT CAG CCC CG -3'; desmin: sense 5'- AAC AGC CTC GGT TCC TTG AG -3' and anti-sense 5'- GAC CTG AGG CTA AAC AGG CG -3' ILK: sense 5'- CAT CAA TGC AGT GAA TGA GC -3'and anti-sense 5'- GAC ATT CCT CAT TGA AGT CC -3'; $\beta$-actin: sense 5'-TCC TTC CTG GGC ATG GAG TC-3', anti-sense 5'-TTC TGC ATC CTG TCG GCA AT3'. The following sequence-specific primers for rat kidney tissue were used: nephrin: sense $5^{\prime}$ - CCC AGG TAC ACA GAG CAC AA -3'and anti-sense 5'- CTC ACG CTC ACA ACC TTC AG -3'; desmin: sense 5'- TGC AGC CAC TCT AGC TCG TA -3' and anti-sense 5'- GAC ATG TCC ATC TCC ACC TG -3' ILK: sense 5'- CAT TGT TGT GAA GGT GCT GA -3'and anti-sense 5'- CAG TGT GTG ATG AGG GTT GG -3'; $\beta$-actin: sense5'- CAG CTG AGA GGG AAA TCG TG -3' anti-sense 5'- CGT TGC CAA TAG TGA TGA CC -3'. Quantitative RT-PCR was performed using the ABI PRISM7500 Sequence Detection System (Applied Biosystems) with SYBR Green Master Mix. Each reaction was amplified in triplicate and ratio results were calculated based on the $2^{-\Delta \Delta \mathrm{CT}}$ method as described according to the previous study [14]. Gene expression was normalized to $\beta$-actin mRNA levels as an endogenous control.

Immunofluorescence analysis

Podocytes under different experimental conditions were fixed for $15 \mathrm{~min}$ at room temperature in $4 \%$ paraformaldehyde, followed by permeabilization with phosphate-buffered saline for 10 min. After blocking with $1 \%$ bovine serum albumin for $1 \mathrm{~h}$, cells were incubated with primary antibodies against desmin (Santa Cruz Biotechnology, Santa Cruz, CA). Negative controls were performed without primary antibody. After washing, the secondary antibody was added and incubated $1 \mathrm{~h}$ at room temperature. Fluorescein(FITC) conjugated secondary antibody (Shanghai PuZhen Biotechnology Co., Ltd.) was added at room temperature for $1 \mathrm{~h}$. Cells were then visualised by fluorescence microscope (BX41, Olympus,Japan).

\section{Statistical analysis}

All data were expressed as means \pm standard deviation (SD). The significance of differences among experimental groups was determined by one-way ANOVA analysis followed by Dunnett's multiple range test. A $P$ value $<0.05$ was considered statistically significant. Statistical analyses were conducted using SPSS 17.0 software.

\section{Results}

Effects of EMO on the expression of nephrin, desmin and ILK in HG-stimulated podocytes

In order to investigate the effects of EMO on HG-induced EMT in podocytes, we examined the epithelial marker nephrin, the mesenchymal marker desmin and ILK. Compared with incubation in NG, exposure to $\mathrm{HG}$ for $24 \mathrm{~h}$ elevated the protein expression of both desmin and ILK while reduced the nephrin protein expression in podocytes. Podocytes treated with EMO showed a significant decrease in desmin and ILK protein expression, as well as an increase in nephrin expression. Likewise, ILK inhibitor QLT0267 also reduced desmin protein expression and elevated nephrin protein expression in HG-stimulated podocytes (Fig. 1). Immunofluorescence analysis further confirmed that HG induced an increment in desmin expression in podocytes. However, the HG-induced increase in desmin expression was alleviated by EMO or QLT0267 treatment (Fig. 2). These results showed that EMO partially restored epithelial marker nephrin expression and inhibited mesenchymal marker desmin and ILK expression in HG-stimulated podocytes.

Effects of EMO on the mRNA expression of nephrin, desmin and ILK in HG-stimulated podocytes

HG for $24 \mathrm{~h}$ significantly increased the mRNA expression of both desmin and ILK while reduced nephrin mRNA expression in podocytes. However, the HG-induced decrease 
Fig. 1. Effects of Emodin (EMO) on nephrin, desmin and ILK expression in HG-stimulated podocytes. Podocytes were exposed to $\mathrm{NG}, \mathrm{HG}, \mathrm{HG}$ with $30 \mu \mathrm{g} / \mathrm{ml}$ of EMO (HG+EMO) and $1 \mu \mathrm{g} / \mathrm{ml}$ of ILK inhibitor QLT0267 (HG+QLT0267) for $24 \mathrm{~h}$. The expression of nephrin, desmin and ILK was examined by Western blot. NG, normal glucose (5 mM glucose ); $\mathrm{HG}$, high glucose (30 mM glucose); HG+EMO, HG with $30 \mu \mathrm{g} / \mathrm{ml}$ of EMO; HG+QLT0267,HG with $1 \mu \mathrm{mol} / \mathrm{ml}$ of QLT0267. Results were expressed as the means $\pm \mathrm{SD}$ (n=4). ${ }^{\text {a }} P<0.05$ vs NG; ${ }^{\text {b }} P<0.05$ vs HG.

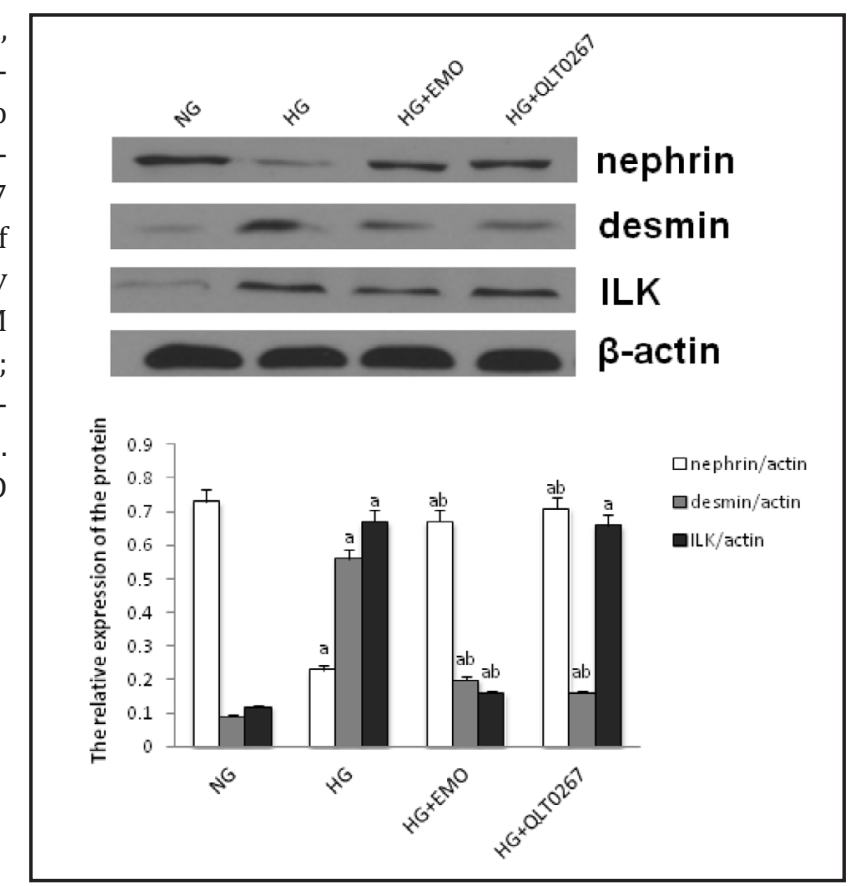

Fig. 2. Effects of Emodin (EMO) on desmin expression detected by immunofluorescence in HG-stimulated podocytes. Podocytes were exposed to $\mathrm{NG}, \mathrm{HG}, \mathrm{HG}$ with $30 \mu \mathrm{g} / \mathrm{ml}$ of EMO (HG+EMO) and $1 \mu \mathrm{g} / \mathrm{ml}$ of ILK inhibitor QLT0267 (HG+QLT0267) for 24 h. NG, normal glucose (5 mM glucose ); HG, high glucose (30 mM glucose); $\mathrm{HG}+\mathrm{EMO}, \mathrm{HG}$ with $30 \mu \mathrm{g} / \mathrm{ml}$ of EMO; $\mathrm{HG}+\mathrm{QLT} 0267, \mathrm{HG}$ with $1 \mu \mathrm{mol} / \mathrm{ml}$ of QLT0267.

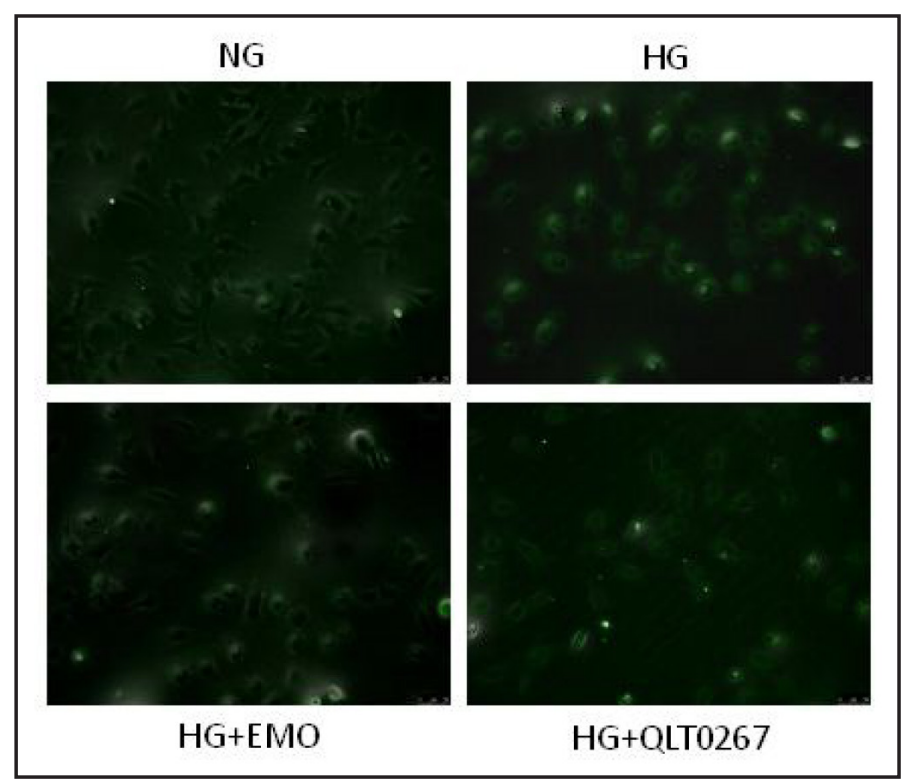

in nephrin mRNA expression was partially restored by EMO treatment (Fig. 3). EMO also significantly reduced desmin and ILK mRNA expression in HG-stimulated podocytes. ILK inhibitor QLT0267 had similar effects on mRNA expression of desmin and nephrin in HGstimulated podocytes.

\section{Effects of EMO on biochemical markers in diabetic rats}

As shown in Figure 4, blood glucose (BG) level was significantly higher in diabetic rats compared with nondiabetic control animals. However, no differences in BG level was observed between EMO treated and untreated STZ-induced diabetic rats (Fig. 4A). Moreover, the STZ-induced diabetic rats showed severe urinary albumin/creatinine ratio (ACR) when compared with the nornmal control rats $(P<0.05)$. However, treatment with EMO or QLT0267 significantly reduced urinary ACR (expressed as mg/g Cr ) in STZ-induced diabetic rats, which indicated that EMO reduced albuminuria similar to QLT0267 (Fig. 4B). 
Fig. 3. Effects of EMO on mRNA expression nephrin, desmin and ILK in HG-stimulated podocytes. Podocytes were exposed to $\mathrm{NG}, \mathrm{HG}$, $\mathrm{HG}$ with $30 \mu \mathrm{g} / \mathrm{ml}$ of EMO (HG+EMO) and $1 \mu \mathrm{g} / \mathrm{ml}$ of ILK inhibitor QLT0267 (HG+QLT0267) for 24 h. The expression of nephrin,desmin and ILK was examined by Real-time quantitative RT-PCR. NG, normal glucose (5 $\mathrm{mM}$ glucose ); $\mathrm{HG}$, high glucose (30 mM glucose); $\mathrm{HG}+\mathrm{EMO}, \mathrm{HG}$ with $30 \mu \mathrm{g} / \mathrm{ml}$ of EMO; HG+QLT0267,HG with $1 \mu \mathrm{mol} / \mathrm{ml}$ of QLT0267. Results were expressed as the means \pm SD $(\mathrm{n}=4)$. ${ }^{\mathrm{a}} P<0.05$ vs NG; ${ }^{\mathrm{b}} P<0.05$ vs HG.

Fig. 4. Effects of EMO on biochemical markers in STZ-induced diabetic rats. EMO and QLT0267 were started at 2 weeks after STZ injection and were administered once daily by oral garage for 12 weeks. Norm, normal control rat; DM, STZ-induced diabetic rat; DM+EMO, DM rats treated with 20 $\mathrm{mg} \cdot \mathrm{kg}^{-1} \cdot \mathrm{d}^{-1}$ of EMO; DM+QLT0267, DM rats treated with $10 \mathrm{mg} \cdot \mathrm{kg}^{-1} \cdot \mathrm{d}^{-1}$ of QLT0267. Results were expressed as the means $\pm \mathrm{SD}(\mathrm{n}=12) .{ }^{\text {a }} P$ $<0.05$ vs Norm group; ${ }^{\mathrm{b}} P<0.05$ vs DM group.

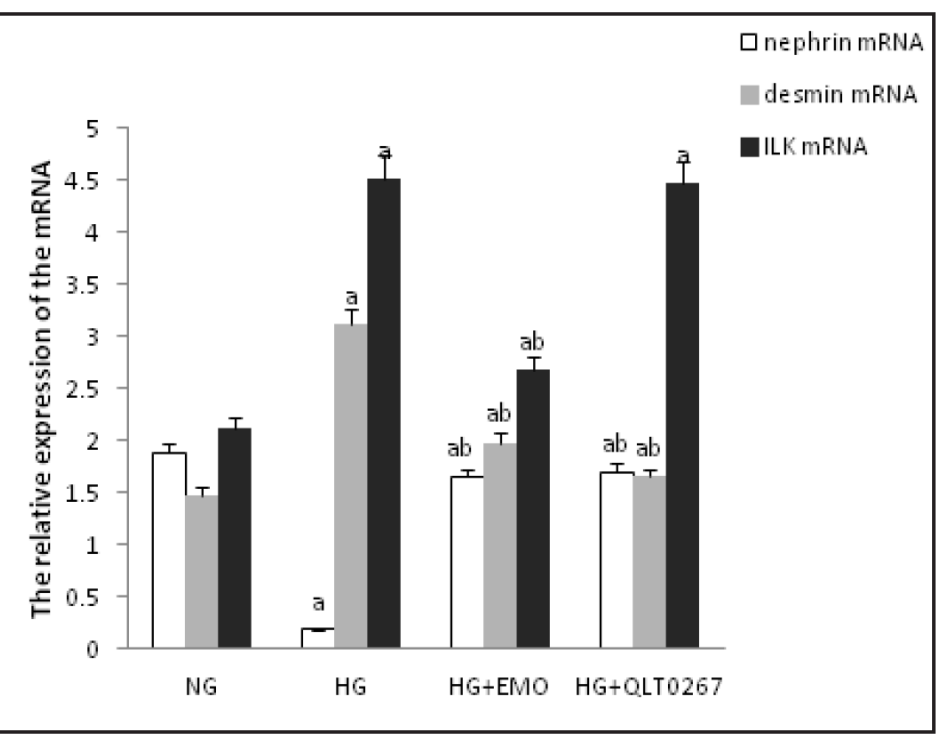

A

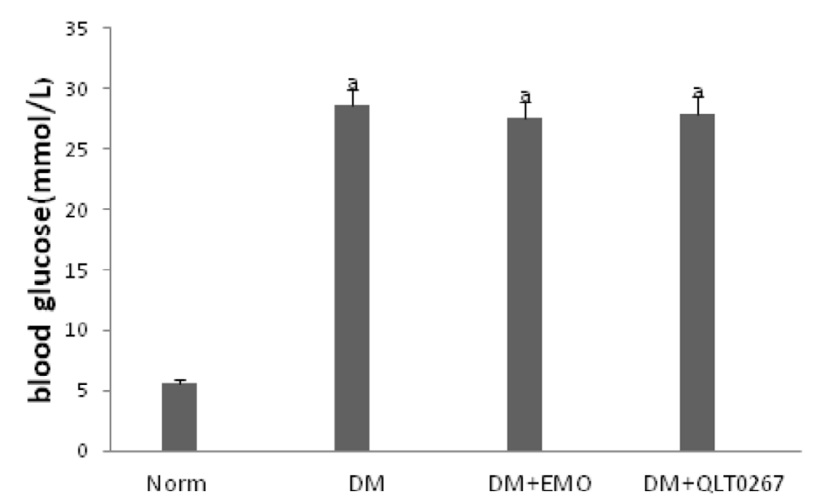

B

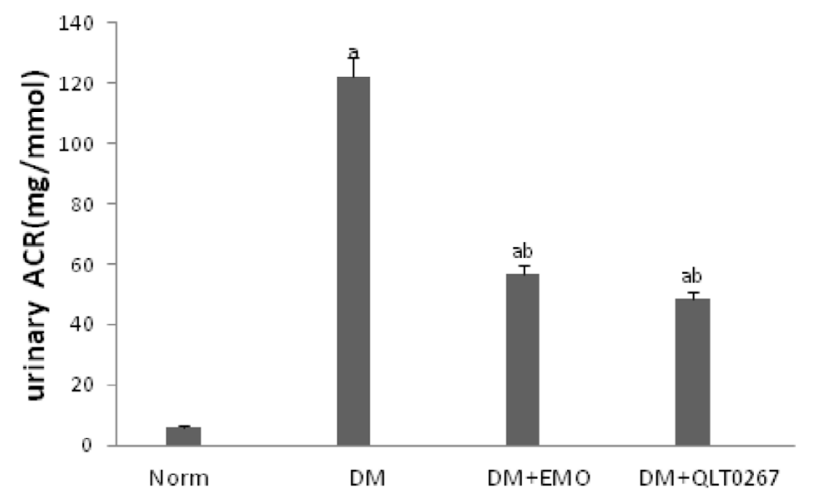

Effects of EMO on renal histopathology and podocyte foot process effacement in diabetic rats.

The STZ-induced diabetic rats showed focal mesangial matrix expansion when compared with normal control rats. However, EMO treatment significantly attenuated 


\section{Cellular Physiology Cell Physiol Biochem 2015;35:1425-1436

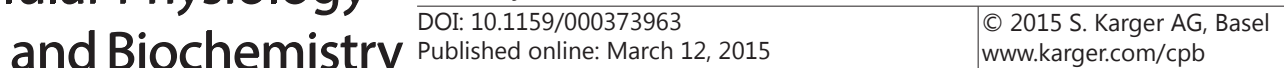

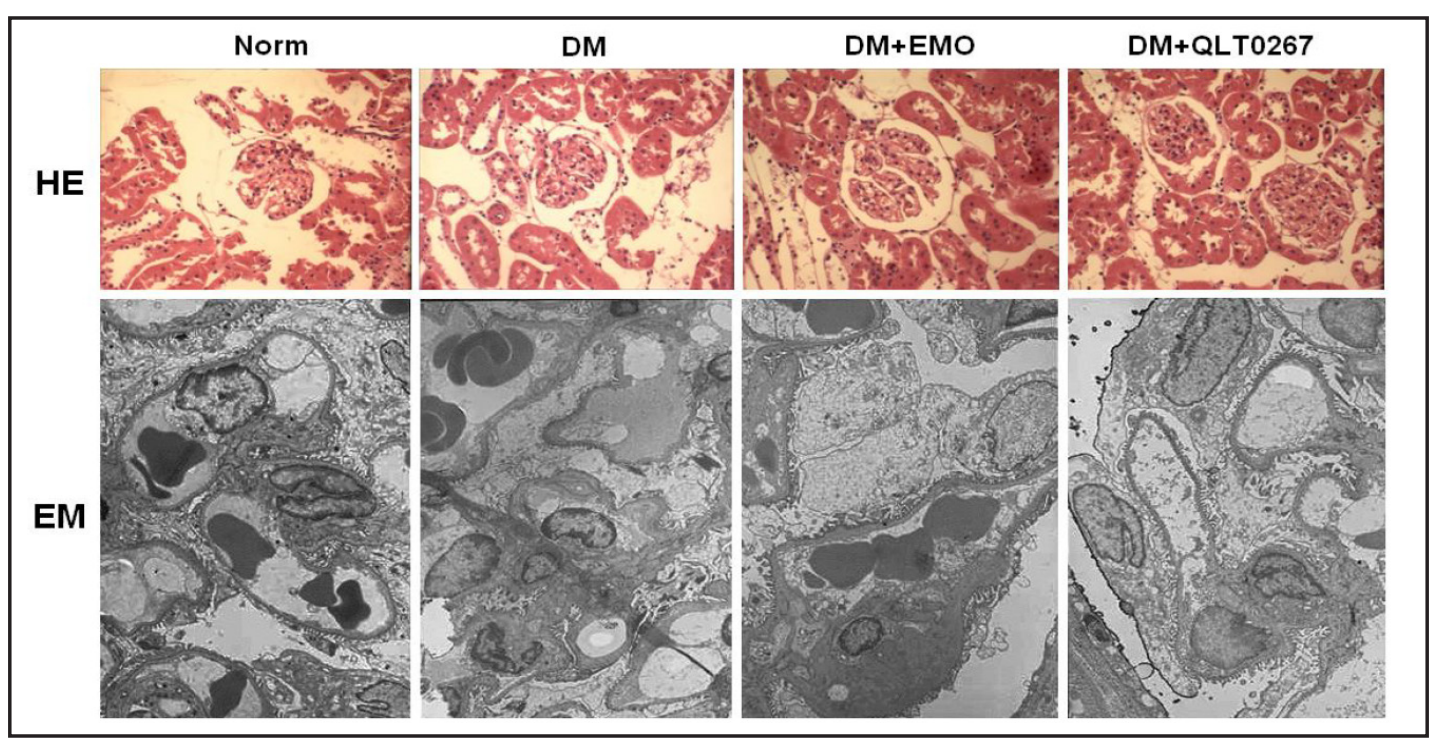

Fig. 5. Effects of EMO on renal histopathology and podocyte foot process effacement in STZ-induced diabetic rats. EMO and QLT0267 were started at 2 weeks after STZ injection and were administered once daily by oral garage for 12 weeks. Norm, normal control rat; DM, STZ-induced diabetic rat; DM+EMO, DM rats treated with $20 \mathrm{mg} \cdot \mathrm{kg}^{-1} \cdot \mathrm{d}^{-1}$ of EMO; DM+QLT0267, DM rats treated with $10 \mathrm{mg} \cdot \mathrm{kg}^{-1} \cdot \mathrm{d}^{-1}$ of QLT0267.

Fig. 6. Effects of EMO on expression of nephrin, desmin and ILK detected by immunohistochemical staining in STZ-induced diabetic rats. Representative nephrin, desmin and ILK immunostaining in the glomeruli of kidney sections (A); Quantitative analyses of changes in expression of nephrin, desmin and ILK (B). EMO and QLT0267 were started at 2 weeks after STZ injection and were administered once daily by oral garage for 12 weeks. Norm, normal control rat; DM, STZ-induced diabetic rat; $\mathrm{DM}+\mathrm{EMO}$, DM rats treated with $20 \mathrm{mg} \cdot \mathrm{kg}^{-}$ ${ }^{1} \cdot \mathrm{d}^{-1}$ of EMO; DM+QLT0267, DM rats treated with $10 \mathrm{mg} \cdot \mathrm{kg}^{-1} \cdot \mathrm{d}^{-1}$ of QLT0267. Results were expressed as the means \pm SD $(n=4)$. ${ }^{\text {a }} P$ $<0.05$ vs Norm group; ${ }^{\mathrm{b}} P<0.05$ vs DM group.

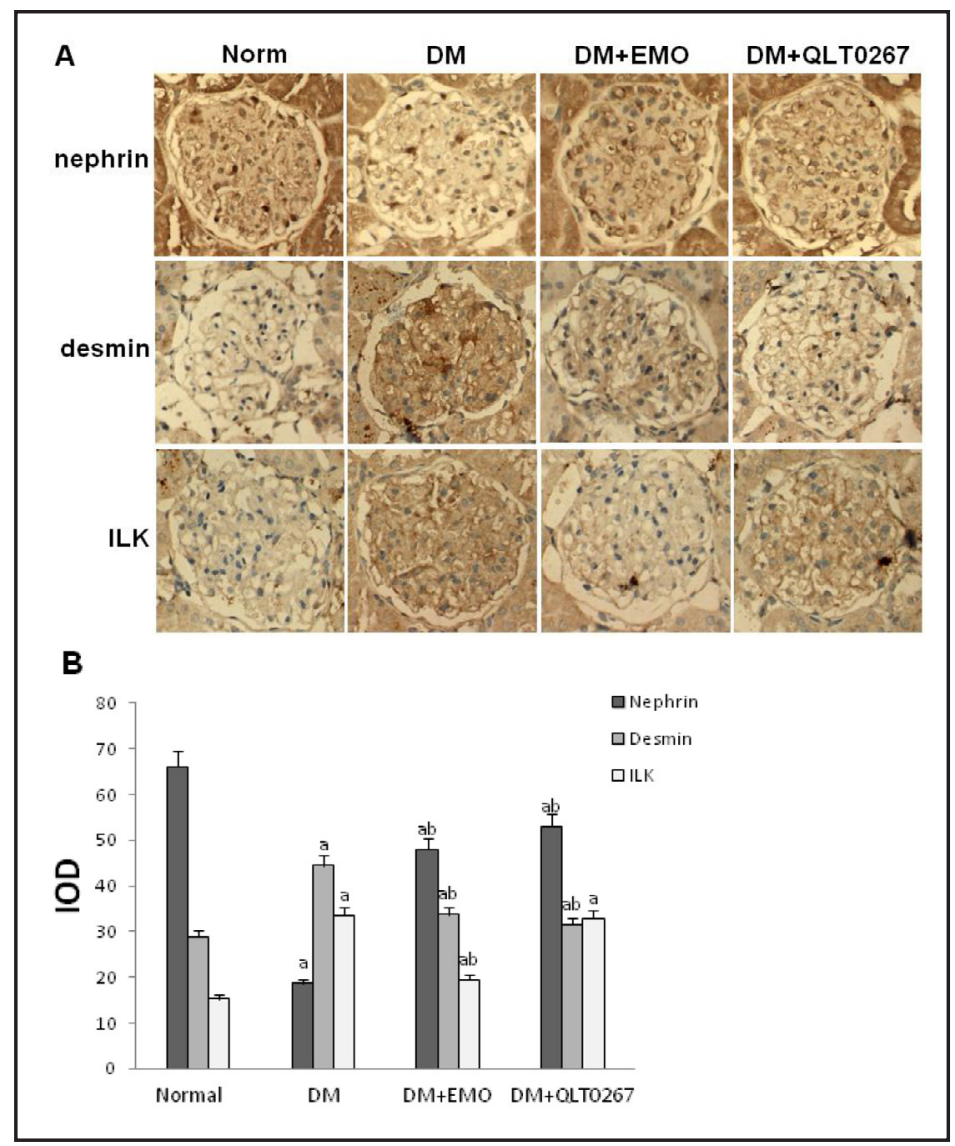

mesangial expansion compared to the untreated STZ-induced diabetic rats (Fig. 5). Moreover, observation of podocyte ultrastructure by electron microscopy (EM) showed apparent podocyte foot process effacement in diabetic rats, whereas the rats treated with EMO revealed a marked decrease in podocyte foot process effacement. Inhibition of ILK with 
Fig. 7. Effects of EMO on protein expression of nephrin, desmin and ILK in STZ-induced diabetic rats. EMO and QLT0267 were started at 2 weeks after STZ injection and were administered once daily by oral garage for 12 weeks. The renal expression of nephrin,desmin and ILK was examined by Western blot. Norm, normal control rat; DM, STZ-induced diabetic rat; DM+EMO, DM rats treated with $20 \mathrm{mg} \cdot \mathrm{kg}^{-1} \cdot \mathrm{d}^{-1}$ of EMO; DM+QLT0267, DM rats treated with $10 \mathrm{mg} \cdot \mathrm{kg}^{-1} \cdot \mathrm{d}^{-1}$ of QLT0267. Results were expressed as the means \pm $\mathrm{SD}(\mathrm{n}=4) .{ }^{\mathrm{a}} \mathrm{P}<0.05$ vs Norm group; ${ }^{\mathrm{b}} P<0.05$ vs DM group.

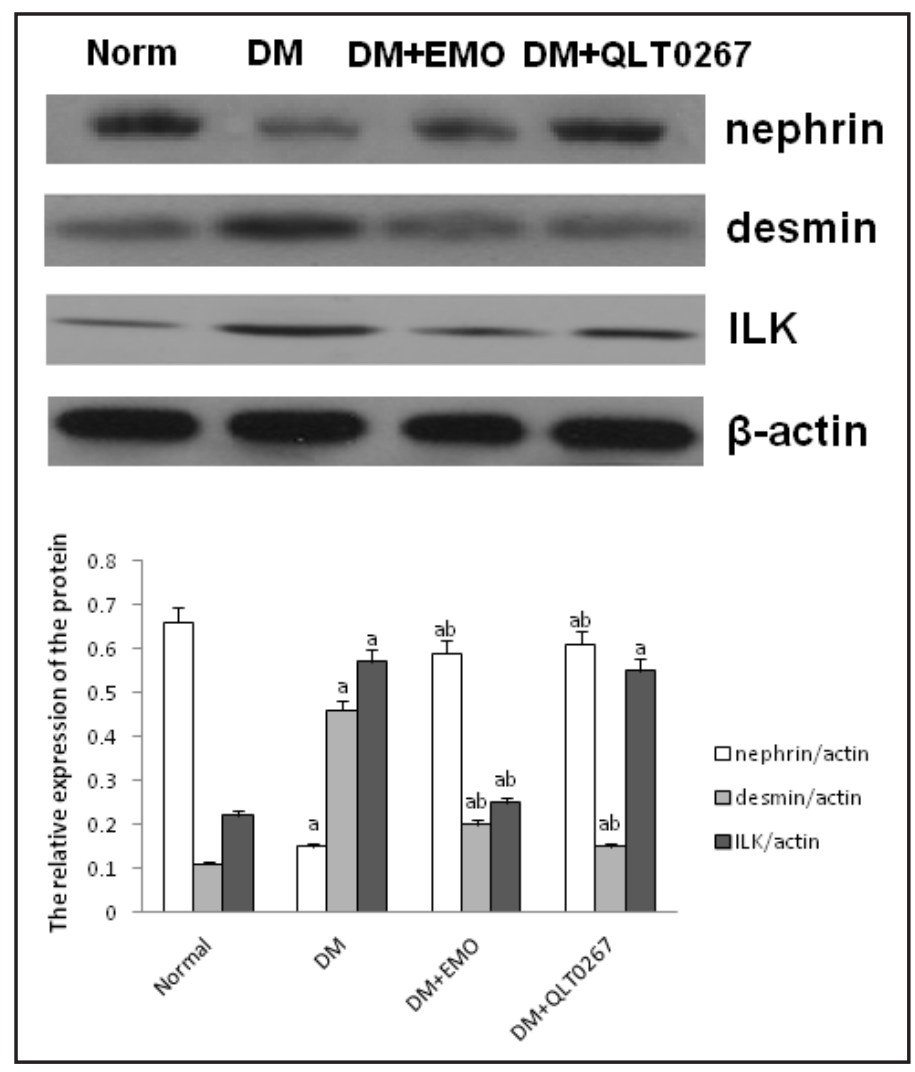

Fig. 8. Effects of EMO on mRNA expression of nephrin, desmin and ILK in STZ-induced diabetic rats. EMO and QLT0267 were started at 2 weeks after STZ injection and were administered once daily by oral garage for 12 weeks. The renal mRNA expression of nephrin, desmin and ILK was examined by Real-time quantitative RT-PCR. Norm, normal control rat; DM, STZ-induced diabetic rat; DM+EMO, DM rats treated with 20 $\mathrm{mg} \cdot \mathrm{kg}^{-1} \cdot \mathrm{d}^{-1}$ of EMO; DM+QLT0267, DM rats treated with $10 \mathrm{mg} \cdot \mathrm{kg}^{-1} \cdot \mathrm{d}^{-1}$ of QLT0267. Results were expressed as the means \pm SD (n $=4) .{ }^{\mathrm{a}} P<0.05$ vs Norm group; ${ }^{\mathrm{b}} P<0.05$ vs DM group.

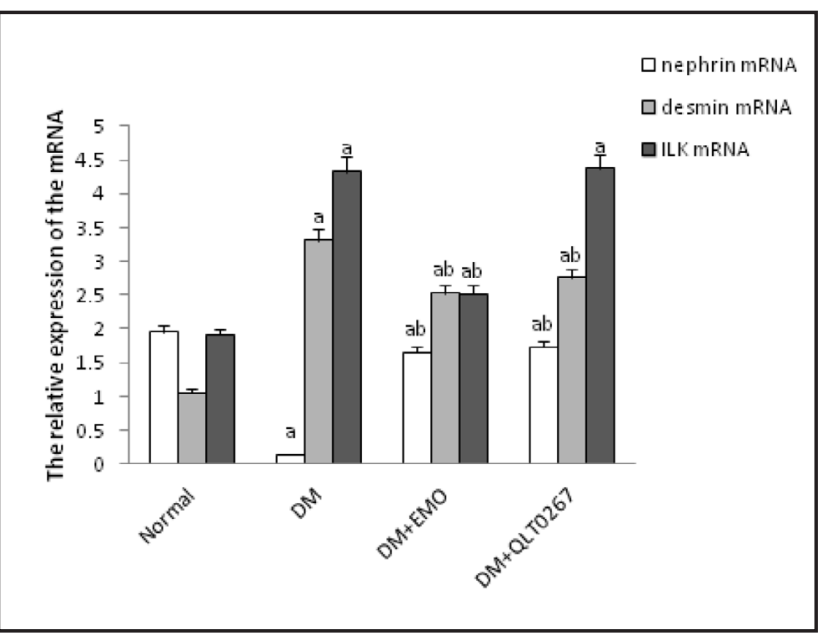

QLT0267 had similar effects on renal histopathology and podocyte foot process effacement to that of EMO (Fig. 5).

\section{Effects of EMO on expression of nephrin, desmin and ILK in diabetic rats}

By immunohistochemical staining, we observed that the expression of desmin and ILK was markedly elevated while the nephrin expression was reduced in the renal tissue from STZ-induced diabetic rats when compared with the normal control rats. However, all of these abnormalities were partially restored by EMO treatment. ILK inhibitor QLT0267 had similar effects on the renal expression of nephrin, desmin and ILK in diabetic rats (Fig. 6). The results of Western blot analysis further confirmed these findings (Fig. 7). The protein expression of desmin and ILK was increased in kidneys from diabetic rats when compared with the normal control rats, which was partially abrogated by treatment with EMO. Moreover, diabetic rats 


\section{Cellular Physiology Cell Physiol Biochem 2015;35:1425-1436 \begin{tabular}{l|l} 
and Biochemistry Published online: March 12, 2015 & $\begin{array}{l}\text { C) 2015 S. Karger AG, Basel } \\
\text { www.karger.com/cpb }\end{array}$ \\
\hline
\end{tabular} \\ Chen et al.: Emodin Ameliorates Podocyte Epithelial-Mesenchymal Transition}

revealed a significant decrease in protein level of nephrin while the rats received EMO showed a marked increase in protein production of nephrin (Fig. 7). Futhermore, changes in mRNA expression of nephrin, desmin and ILK were quantified by real-time quantitative RTPCR. Similarly, renal cortical mRNA expression of desmin and ILK were up-regulated while the nephrin expression was down-regulated in diabetic rats, which was partially attenuated by treatment with EMO (Fig. 8). The above results demonstrated that the protective effect of EMO on podocyte EMT and subsequent podocyte depopulation in diabetic rats was associated with the inhibition of ILK and desmin as well as upregulatiotion of nephrin.

\section{Discussion}

Diabetic kidney disease (DKD) is becoming a major cause of ESRD. However, there is no completely effective treatment at present. It is urgent to find the novel treatments to prevent DKD. Podocytes are highly differentiated epithelial cells located in the the outer surface of the glomerular basement membrane (GBM) and play a key role in maintaining the structure and function of the glomerular filtration barrier. Recent studies have increasingly demonstrated that podocyte injury plays an important role in the development of $\operatorname{DKD}[15,16]$ and emerging evidence suggests that DKD is more a "podocyte disease" [17]. Accumulating evidences suggest that high glucose environment, such as the one observed in diabetes, could be involved in podocytes EMT [18-20]. In-vivo study further confirmed that podocyte EMT could be a potential pathway leading to proteinuria in DKD [21]. Therefore, EMT is recognized to play a crucial role in the development of DKD and targeting inhibition of EMT may provide new therapeutic approaches for DKD. In this study, we firstly reported that EMO, a major component of Rheum palmatum, ameliorated podocyte EMT under diabetic condition in-vitro and in-vivo. This conclusion was based upon the following findings: (i) EMO significantly inhibited ILK and desmin expression and partially restored nephrin expression in HG-stimulated podocytes; (ii) Treatment with EMO repressed renal ILK and desmin expression, preserved nephrin expression in diabetic rats; (iii) EMO significantly reduced albuminuria in diabetic rats. Therefore, EMO might be a novel drug to reverse HGinduced podocyte EMT, which provided a potential new therapy in the treatment of DKD and other renal diseases affecting podocytes.

Accumulating evidences have suggested that podocytes undergo EMT in many diseases including DN, which leads to proteinuria and ultimately results in kidney fibrosis [21-24]. Podocytes epithelial markers, such as nephrin and zonula occludens-1, are lost under diabetic condition, while mesenchymal cell markers, such as $\alpha$-SMA and fibronectin are expressed, leading to podocyte dysfunction and proteinuria [25-28]. Recent study demonstrated that HG promoted podocyte EMT, as shown by decreased nephrin expression, and increased $\alpha$-SMA expression [29]. In-vivo study further confirmed that in addition to loss of nephrin and zonula occludens-1, mesenchymal markers such as desmin, fibroblast-specific protein-1, and matrix metalloproteinase- 9 could be observed in glomerular podocytes of diabetic nephropathy [21]. As nephrin is one of epithelial markers, the decreasing nephrin expression may be responsible for the increasing albuminuria in the early stage of DKD [30]. Desmin, an intermediate filament protein, has long been suggested as a podocyte injury indicator. As an EMT key marker, the expression of desmin was specifically localized in glomerular podocytes [21]. In this study, HG increased podocyte desmin expression while decreased nephrin expression in-vitro and iv-vivo, which was in agreement with above studies. However, EMO significantly inhibited desmin expression and partially restored nephrin expression in HG-stimulated podocytes. These in-vitro observations were further confirmed in-vivo. Treatment with EMO for 12 weeks repressed renal ILK and desmin expression, preserved nephrin expression, as well as ameliorated albuminuria in STZ-induced diabetic rats. These results clearly demonstrated that EMO ameliorated glucose-induced EMT and subsequent podocyte dysfunction partly through desmin inhibition as well as nephrin upregulatiotion. These findings might provide a potential novel therapeutic option for DKD. 
To reveal the mechanisms underlying the action of EMO on HG induced-podocyte EMT, we investigated the effects of EMO and ILK inhibitor QLT0267 on expression of ILK, desmin and nephrin in HG-stimulated podocytes and diabetic rats. ILK expression has been demonstrated to be dramatically elevated in diabetic glomeruli [31]. Our previous studies have shown that HG elevated ILK activity in cultured mouse podocytes [32] and renal ILK was activated in STZ-induced diabetic rats [33]. Recent studies have demonstrated that TGF- $\beta 1$ and high glucose induced podocytes EMT [21, 34, 35] and that ILK was a crucial intracellular mediator in this cellular event, which was shown by the fact that inhibition of ILK with smallmolecular inhibitor QLT-0267 attenuated podocyte EMT and proteinuria. Importantly, the inhibitor QLT-0267 exited no adverse effect on normal kidney structure and function [35]. Consistent with these results, the present study showed that high glucose induced podocyte EMT and ILK overexpression. Previous study found that ILK upregulation and nephrin downregulation disrupted the balance of the ternary complex, which might be responsible for EMT [36]. In this study, EMO significantly inhibited desmin expression and partially restored nephrin expression in HG-stimulated podocytes. Furthermore, EMO inhibited the overexpression of ILK and ameliorated the EMT of podocytes. Thus, the regulatory effect of EMO on ILK is likely to be accountable for its protective effects against podocyte EMT.

It was reported that EMO inhibited cell proliferation and fibronectin expression through p38MAPK pathway in rat mesangial cells cultured under high glucose [37]. EMO was shown to have antiinflammatory effects [38] and EMO also attenuated glucose-induced matrix synthesis in human peritoneal mesothelial cells [39]. Recent study have demonstrated that EMO reduced TGF- $\beta 1$ and FN overexpression by inhibition of NF- $\kappa B$ activation, suggesting that EMO-mediated suppression of the NF- $\kappa$ B pathway could protect against DKD [40]. However, the effects of EMO on podocytes EMT in rats with DKD have not been investigated yet. In this study, we investigated the protective effects of EMO on podocyte EMT under diabetic condition in-vitro and in-vivo and found that HG increased podocyte ILK and desmin expression while decreased nephrin expression, which were partially restored by EMO treatment. In-vivo study further confirmed that EMO repressed renal ILK and desmin expression, preserved nephrin expression, as well as ameliorated albuminuria in diabetic rats. These results indicated that EMO could be a potential novel drug for the treatment of DKD.

There was limitation in this study. We only measured the blood glucose level in rats, and the effect of EMO on serum insulin level should be further investigated in the future study.

In conclusion, our study clearly demonstrated that EMO ameliorated HG-induced EMT and sudsequent podocyte dysfunction through nephrin upregulation, as well as desmin and ILK inhibition in-vitro and in-vivo. These findings might provide a potential new therapeutic option for DKD and other renal diseases affecting podocytes.

\section{Acknowledgments}

This work was supported by grants from National Natural Science Foundation of China (81270824 to Niansong Wang and 81000305 to Dingkun Gui) and Traditional Chinese Medicine Foundation of Shanghai Municipal Commission of Health and Family Planning (2014JP018A). This work was funded by Key Disciplines Group Construction Project of Pudong Health Bureau of Shanghai (PWZxq2014-07) and Joint Research Project of Pudong Health Bureau of Shanghai (PW2013D-5). This work was also supported by grants from Medical-Engineering Cross Project of Shanghai Jiao Tong University (YG2014MS20) and Outstanding Young Talents Program in East campus, Shanghai Jiao Tong University Affiliated Sixth People's Hospital.

\section{Disclosure Statement}

The authors have declared that no competing interests exist.

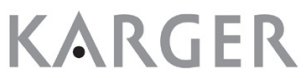




\section{Cellular Physiology Cell Physiol Biochem 2015;35:1425-1436 \begin{tabular}{l|l} 
and Biochemistry $\begin{array}{l}\text { Dublished online: March 12, } 2015 \\
\text { Po 2015 S. Karger AG, Basel } \\
\text { www.karger.com/cpb }\end{array}$ \\
\hline
\end{tabular} \\ Chen et al.: Emodin Ameliorates Podocyte Epithelial-Mesenchymal Transition}

\section{References}

1 USRDS: The United States Renal Data System. Am J Kidney Dis 2003;42:1-230.

2 Pagtalunan ME, Miller PL, Jumping-Eagle S, Nelson RG, Myers BD, Rennke HG, Coplon NS, Sun L, Meyer TW: Podocyte loss and progressive glomerular injury in type II diabetes. J Clin Invest 1997;99:342-348.

3 Yamaguchi Y, Iwano M, Suzuki D, Nakatani K, Kimura K, Harada K, Kubo A, Akai Y, Toyoda M, Kanauchi M, Neilson EG, Saito Y:Epithelial-mesenchymal transition as an explanation for podocyte depletion in diabetic nephropathy. Am J Kidney Dis 2009;54:653-664.

4 Li Y, Kang YS, Dai C, Kiss LP, Wen X, Liu Y: Epithelial-to-mesenchymal transition is a potential pathway leading to podocyte dysfunction and proteinuria. Am J Pathol 2008;172:299-308.

5 Liu Y. New insights into epithelial-mesenchymal transition in kidney fibrosis. J Am Soc Nephrol 2010;21:212-222.

6 Vogelmann SU,Nelson WJ,Myers BD,Lemley KV:Urinary excretion of viable podocytes in health and renaldisease. Am J Physiol Renal Physiol 2003;285:F40-48.

7 Petermann AT, Pippin J, Krofft R, Blonski M, Griffin S, Durvasula R, Shankland SJ: Viable podocytes detach in experimental diabetic nephropathy:potential mechanism underlying glomerulosclerosis. Nephron Exp Nephrol 2004;98:e114-123.

8 Waly MI, Ali BH, Al-Lawati I, Nemmar A:Protective effects of emodin against cisplatin-induced oxidative stress in cultured human kidney (HEK 293) cells. J Appl Toxicol 2013;33:626-630.

9 Lei Q, Qiang F, Chao D, Di W, Guoqian Z, Bo Y, Lina Y: Amelioration of hypoxia and LPS-induced intestinal epithelial barrier dysfunction by emodin through the suppression of the NF- $\mathrm{KB}$ and HIF-1 $\alpha$ signaling pathways. Int J Mol Med 2014;34:1629-1639.

10 Wu Z, Chen Q Ke D, Li G, Deng W: Emodin protects against diabetic cardiomyopathy by regulating the AKT/ GSK-3 $\beta$ signaling pathway in the rat model. Molecules 2014;19:14782-14793.

11 Wang J, Huang H, Liu P, Tang F, Qin J, Huang W, Chen F, Guo F, Liu W, Yang B: Inhibition of phosphorylation of p38 MAPK involved in the protection of nephropathy by emodin in diabetic rats. Eur J Pharmacol 2006;553:297-303.

12 Gui D, Guo Y, Wang F, Liu W, Chen J, Chen Y, Huang J, Wang N: Astragaloside IV, a novel antioxidant, prevents glucose-induced podocyte apoptosis in-vitro and in-vivo. PLoS One 2012;7:e39824.

13 Mundel P, Reiser J, Zúñiga Mejía Borja A, Pavenstädt H, Davidson GR, Kriz W, Zeller R: Rearrangements of the cytoskeleton and cell contacts induce process formation during differentiation of conditionally immortalized mouse podocytes cell lines. Exp Cell Res 1997;236:248-258.

14 Livak KJ, Schmittgen TD: Analysis of relative gene expression data using real-time quantitative PCR and the $2^{-\Delta \Delta C T}$ method. Methods 2001;25:402-408.

15 Anil Kumar P, Welsh GI, Saleem MA, Menon RK: Molecular and cellular events mediating glomerular podocyte dysfunction and depletion in diabetes mellitus. Front Endocrinol (Lausanne) 2014;5:151.

16 Wang L, Tang Y, Eisner W, Sparks MA, Buckley AF, Spurney RF: Augmenting podocyte injury promotes advanced diabetic kidney disease in Akita mice. Biochem Biophys Res Commun 2014;444:622-627.

17 Kim JJ, Li JJ, Jung DS, Kwak SJ, Ryu DR, Yoo TH, Han SH, Choi HY, Kim HJ, Han DS, Kang SW: Differential expression of nephrin according to glomerular size in early diabetic kidney disease. J Am Soc Nephrol 2007;18:2303-2310.

18 Lv Z, Hu M, Zhen J, Lin J, Wang Q Wang R: Rac1/pak1 signaling promotes epithelial-mesenchymal transition of podocytes in-vitro via triggering beta-catenin transcriptional activity under high glucose conditions. Int J Biochem Cell Biol 2013;45:255-264.

19 Wang C, Liu X, Ke Z, Tang Y, Li CC, Li CM, Ye Z, Zhang J, Lou T: Mesangial medium from IgA nephropathy patients induces podocyte epithelial-to-mesenchymal transition through activation of the phosphatidyl inositol-3-kinase/akt signaling pathway. Cell Physiol Biochem 2012;29:743-752.

20 Boini KM, Xia M, Xiong J, Li C, Payne LP, Li PL: Implication of cd38 gene in podocyte epithelial-tomesenchymal transition and glomerular sclerosis. J Cell Mol Med 2012;16:1674-1685.

21 Li Y, Kang YS, Dai C, Kiss LP, Wen X, Liu Y: Epithelial-to-mesenchymal transition is a potential pathway leading to podocyte dysfunction and proteinuria. Am J Pathol 2008;172:299-308

22 Yamaguchi Y, Iwano M, Toyoda M, Suzuki D, Nakatani K, Kimura K, Harada K, Kubo A, Akai Y, Toyoda M, Kanauchi M, Neilson EG, Saito Y: Epithelial-mesenchymal transition as an explanation for podocyte depletion in diabetic nephropathy. Am J Kidney Dis 2009;54:653-664. 


\section{Cellular Physiology Cell Physiol Biochem 2015;35:1425-1436 \begin{tabular}{l|l|l|}
\cline { 2 - 2 } DOI: 10.1159/000373963 & O 2015 S. Karger AG, Basel \\
\hline
\end{tabular}

23 Reidy K, Susztak K: Epithelial-mesenchymal transition and podocyte loss in diabetic kidney disease. Am J Kidney Dis 2009;54:590-593.

24 Liu Y: New insights into epithelial-mesenchymal transition in kidney fibrosis. J Am Soc Nephrol 2010;21:212-222.

25 Rincon-Choles H, Vasylyeva TL, Pergola PE, Bhandari B, Bhandari K, Zhang JH, Wang W, Gorin Y, Barnes JL, Abboud HE: Zo-1 expression and phosphorylation in diabetic nephropathy. Diabetes 2006;55:894-900.

26 Xu ZG, Ryu DR, Yoo TH, Jung DS, Kim JJ, Kim HJ, Choi HY, Kim JS, Adler SG, Natarajan R, Han DS, Kang SW: P-cadherin is decreased in diabetic glomeruli and in glucose-stimulated podocytes in-vivo and in-vitro studies. Nephrol Dial Transplant 2005;20:524-531.

27 Tesar V, Zima T: Recent progress in the pathogenesis of nephrotic proteinuria. Crit Rev Clin Lab Sci 2008;45:139-220.

28 Jefferson JA, Shankland SJ, Pichler RH: Proteinuria in diabetic kidney disease: A mechanistic viewpoint. Kidney Int 2008;74:22-36.

29 Guo J, Xia N, Yang L, Zhou S, Zhang Q, Qiao Y, Liu Z: GSK-3 $\beta$ and Vitamin D Receptor are Involved in $\beta$-Catenin and Snail Signaling in High Glucose-Induced EpithelialMesenchymal Transition of Mouse Podocytes. Cell Physiol Biochem 2014;33:1087-1096.

30 Kim JJ, Li JJ, Jung DS, Kwak SJ, Ryu DR, Yoo TH, Han SH, Choi HY, Kim HJ, Han DS, Kang SW: Differential expression of nephrin according to glomerular size in early diabetic kidney disease. J Am Soc Nephrol 2007;18:2303-2310.

31 Guo L, Sanders PW, Woods A, Wu C : The distribution and regulation of integrin-linked kinase in normal and diabetic kidneys. Am J Pathol 2001;159:1735-1742.

32 Chen J, Gui D, Chen Y, Mou L, Liu Y, Huang J: Astragaloside IV improves high glucose-induced podocyte adhesion dysfunction via $\alpha 3 \beta 1$ integrin upregulation and integrin-linked kinase inhibition. Biochem Pharmacol 2008;76:796-804.

33 Chen Y, Gui D, Chen J, He D, Luo Y, Wang N: Down-regulation of PERK-ATF4-CHOP pathway by Astragaloside IV is associated with the inhibition of endoplasmic reticulum stress-induced podocyte apoptosis in diabetic rats. Cell Physiol Biochem 2014 33:1975-1987.

34 Yamaguchi Y, Iwano M, Suzuki D, Nakatani K, Kimura K, Harada K, Kubo A, Akai Y, Toyoda M, Kanauchi M, Neilson EG, Saito Y:Epithelialmesenchymal transition as a potential explanation for podocyte depletion in diabetic nephropathy. Am J Kidney Dis 2009;54:653-664.

35 Kang YS, Li Y, Dai C, Kiss LP, Wu C, Liu Y: Inhibition of integrin-linked kinase blocks podocyte epithelialmesenchymal transition and ameliorates proteinuria. Kidney Int 2010;78:363-373.

36 Dai C, Stolz DB, Bastacky SI, St-Arnaud R, Wu C, Dedhar S, Liu Y: Essential role of integrin-linked kinase in podocyte biology: Bridging the integrin and slit diaphragm signaling. J Am Soc Nephrol 2006;17:21642175.

37 Li X, Liu W, Wang Q, Liu P, Deng Y, Lan T, Zhang X, Qiu B, Ning H, Huang H: Emodin suppresses cell proliferation and fibronectin expression via p38MAPK pathway in rat mesangial cells cultured under high glucose. Mol Cell Endocrinol 2009;307:157-162.

38 Chang CH, Lin CC, Yang JJ, Namba T, Hattori M: Anti-inflammatory effects of emodin from ventilago leiocarpa. Am J Chin Med 1996;24:139-142.

39 Chan TM, Leung JK, Tsang RC, Liu ZH, Li LS, Yung S: Emodin ameliorates glucose-induced matrix synthesis in human peritoneal mesothelial cells. Kidney Int 2003;64:519-533.

40 Yang J, Zeng Z, Wu T, Yang Z, Liu B, Lan T: Emodin attenuates high glucose-induced TGF- $\beta 1$ and fibronectin expression in mesangial cells through inhibition of NF- $\kappa B$ pathway. Exp Cell Res 2013;319:3182-3189. 